Man and Nature

L'homme et la nature

\title{
La Visée historiographique de Charlevoix d'après ses « Liste et examen des auteurs consultés »
}

\section{Réal Ouellet}

Volume 1, 1982

URI : https://id.erudit.org/iderudit/1011799ar

DOI : https://doi.org/10.7202/1011799ar

Aller au sommaire du numéro

Éditeur(s)

Canadian Society for Eighteenth-Century Studies / Société canadienne d'étude du dix-huitième siècle

ISSN

0824-3298 (imprimé)

1927-8810 (numérique)

Découvrir la revue

Citer cet article

Ouellet, R. (1982). La Visée historiographique de Charlevoix d'après ses « Liste et examen des auteurs consultés ». Man and Nature / L'homme et la nature, 1, 153-163. https://doi.org/10.7202/1011799ar

Copyright (C Canadian Society for Eighteenth-Century Studies / Sociéte canadienne d'étude du dix-huitième siècle, 1982
Ce document est protégé par la loi sur le droit d'auteur. L'utilisation des services d'Érudit (y compris la reproduction) est assujettie à sa politique d'utilisation que vous pouvez consulter en ligne.

https://apropos.erudit.org/fr/usagers/politique-dutilisation/ 


\section{La Visée historiographique de Charlevoix d'après ses "Liste et examen des auteurs consultés"}

Je mettrai à la tête de chaque Histoire un Catalogue exact de tous les Auteurs, qui auront écrit sur le même sujet, ne l'eussent-ils fait qu'en passant, pourvû que ce qu'ils en ont dit, mérite qu'on y fasse quelque attention. Je marquerai en même tems les secours, que j'aurai tirés de chacun, et les raisons, que j'aurai euës de les suivre, ou de m'en écarter; en quoi je tâcherai de faire en sorte, qu'aucune prévention, ni aucun autre intérêt, que celui de la vérité, ne conduise ma plume.

... il étoit bien temps de rendre ce service au Public, tandis que nous avons encore des regles certaines de critique pour distinguer les Pieces légitimes et authentiques, de ce nombre prodigieux d'Ecrits hazardés, dont la plûpart altérent la vérité jusqu'au point de la rendre méconnoissable, et qui en feroient enfin perdre absolument la trace, si on laissoit aller le débordement plus loin. ${ }^{1}$

Le sort réservé à l'Histoire et description générale de la Nouvelle-France est paradoxal. Elle n'a été rééditée en français qu'en 1976 (Montréal; Elysée), et on la cite beaucoup moins que les Relations des Jésuites, Champlain ou même Sagard; et pourtant, elle constitue non seulement la première histoire d'envergure de la Nouvelle-France, mais encore elle a donné à notre histoire sa forme quasi définitive. On a souvent cité cette phrase de Fr.-X. Garneau:

Notre histoire qui n'était avant lui qu'une oeuvre imparfaite a pris sous sa plume les proportions, l'ordre et le développement d'une histoire en forme. $^{2}$

Proportion, ordre, développement; ces mots, qui pourraient être de Charlevoix, renvoient à la double opération du travail historique: sélection des éléments diégétiques et mise en ordre dans une structure narrative et explicative. Dans cette optique, le jugement de Garneau me paraît très juste: de Garneau lui-même jusqu'à Groulx tout au moins, l'histoire du Canada semble avoir emprunté à Charlevoix un cadre global, une forme canonique, tant pour le choix des matériaux que pour le développement du commentaire et l'ordre du récit.

Mais chercher la visée historiographique de Charlevoix dans sa bibliographie critique peut ressembler davantage à une gageure qu'à une 
entreprise sérieuse. Je ne vous cacherai pas le caractère insolite de cette hypothèse de travail. A défaut de toucher l'essentiel peut-être, elle me permettra de questionner d'une manière différente un discours historiographique important du Siècle des Lumières. Un discours, qui, se situant en marge et à la suite d'autres écrits, vise un objectif à double volet cumulatif et normatif - : tout dire de l'essentiel et bien dire la vérité.

\section{L'Histoire comme anti-roman}

Une lecture superficielle de la "Liste des auteurs" dégage une première ligne de force importante: l'insistance sur l' "Histoire suivie et complette," par opposition à l'"Histoire fort détaillée" de la colonie qu'avaient laissée les Jésuites avec leurs Relations. L'Histoire suivie hiérarchise les faits, leur donne liaison et vectorialité, dégage les causes et l'enchaînement, identifie les acteurs des événements; l'histoire détaillée, collée au quotidien, rend le discontinu, la poussière de mille gestes ponctuels, recueille les matériaux sur lesquels l'histoire suivie sera fondée. L'histoire suivie met de l'ordre dans une profusion regardée comme une espèce d'organisme vivant qui menace de s'amplifier au point de "noyer" l'histoire même et de rendre incompréhensible la logique des événements. Non maîtrisée, cette profusion devient fable proliférante occupant tout l'espace historique - comme le cadavre de l'Amédée d'Ionesco finit par occuper tout l'espace scénique. A trois reprises au moins, Charlevoix affirme préférer le vide ou l'exiguïté de la connaissance à la profusion fabuleuse. Les premiers auteurs, "par cela même qu'ils nous apprenoient très-peu de choses," "ne pouvoient pas nous jetter dans de grandes erreurs." On comprend au nom de quel principe un Lahontan est avec violence désavoué: il confond "tellement" "le vray avec le faux," qu'il plonge tout dans l'incertitude. Car en histoire, la fable ou le roman - cet adroit mélange de vérité et de mensonge, comme on disait fréquemment alors - est pire que l'ognorance. Dans son "Avertissement," Charlevoix reproche à Koempfer d'avoir "défiguré" les "trois ou quatre faits historiques" à quoi se réduit son Histoire de Saint-Domingue: "Koempfer en a fait un Roman, où la vraisemblance n'est pas même gardée."

Rappelons au passage qu'un autre Jésuite français, le P. Porée, publia en 1736 un violent réquisitoire contre le roman: De libris qui vulgo dicuntur Romanses; un an auparavant, le $\mathrm{P}$. Bougeant, qui s'intéressa à l'âme des bêtes, publia son Prince Fan-Féredin au royaume de Romancie (XII-275pp.), parodie, qui relève sur le mode humoristique tous les clichés du roman.

La prolifération fabuleuse aura son exacte correspondance dans l'écriture: on pourrait regarder la "liberté de plume" reprochée à Hennepin et Lahontan surtout comme une manifestation stylistique de cette abondance trompeuse, mais c'est dans l'“enflure" verbale, le "style de déclamation" qu'on la retrouvera le mieux: assez curieusement, c'est à deux représentants de l'ordre mendiant, de l'ordre "pauvre", les Récollets Leclercq et Hennepin encore, que sont accolées ces qualifications comme si la pauvreté affirmée de l'extérieur était compensée par la boursouflure langagière. Faut-il encore rattacher à cette prolifération dangereuse les "indécences" et les "libertés" reprochées à Lahontan et Hennepin encore une fois? J'y serais porté, surtout si on les range sous la catégorie du désordre où entrerait encore le style 
"barbare" et "embarrassé" (Lahontan), par opposition au style "simple et naturel" d'un Champlain, par exemple. Car le bien écrire se confond avec la simplicité (Mgr de Saint-Vallier): "Le style de ces relatons (celles des Jésuites) est extrémement simple." On aurait tort de voir toutefois en Charlevoix un adepte de l'ascétisme historique: il aime les amples portraits, les détails nombreux, l'accumulation des questions hypothétiques. Dans le corps de son récit, il ne veut rien sacrifier de la prodigieuse richesse de l'événement, il accueille avec empressement le "curieux", l'“intéressant", l'énigmatique. Je n'en fournirai ici que deux exemples, puisqu'ils ne touchent pas directement à mon propos:

1. Les "Hommes monstrueux" dont on parle dès les pages 15 à 20 du Livre I. Charlevoix, qui écrit de Champlain: "On ne peut lui reprocher qu'un peu trop de credulité pour des contes", s'attarde à parler de "choses extraordinaires", non dénuées de "merveilleux" et "qui ne sont pas tout-à-fait indignes de l'attention des personnes curieuses" (pp. 15-16). Par exemple, des "Hommes d'une grandeur et d'une grosseur monstrueuses, qui rendoient leurs excremens par la bouche, et urinoient par-dessous l'épaule," ou encore ce "Peuple tout noir" aux "cheveux droits blancs" du Labrador ou bien ces Pygmées qui "n'ont pas plus de trois pieds de haut, et sont d'une extrème grosseur." Avant de "mettre fin à cette digression," Charlevoix s'interroge en des termes peut-être un peu surprenants pour un Jésuite cultivé et orthodoxe:

Qui peut s'assûrer de connoître tous les caprices et tous les mysteres de la Nature? On sçait combien l'imagination des Meres a de pouvoir sur le fruit qu'elles portent. L'experience, le témoignage même de l'Ecriture, en sont des preuves sans réplique: ajoûtons à cela les figures bizarres, où certaines Nations trouvent une beauté, dont elles sont si jalouses, qu'on y met les corps des Enfans à la torture pour achever ce que l'imagination des Meres n'a pû finir, et l'on comprendra sans peine qu'il peut y avoir des Hommes assez differens des autres pour donner lieu à certaines gens, qui saisissent vivement les objets, et ne se donnent pas le tems d'examiner les choses, de faire des contes absurdes, qui ne sont pourtant pas sans quelque réalité. Je reviens à mon Histoire.

"Je reviens à mon Histoire," conclut Charlevoix, conscient d'avoir cédé à la "séduction de l'étrange" et à sa propension à formuler des hypothèses lorsque celles-ci ne mettent pas en danger la belle ordonnance logique de l'enchaînement événementiel.

2. En étudiant de près certains passages de l'Histoire et description, j'avais relevé un élément de stratégie narrative visant à dramatiser un événement important. Par exemple, le récit de l'attaque de Phips devant Québec est encadré par deux zones d'ombre laissées au début et à la fin. La première a pour objet la culpabilité plus ou moins grande du Gouverneur de Plaisance que les Anglais venaient de prendre; la seconde pèse les différents témoignages touchant la mésentente de certaines tribus indiennes avec les Anglais et qui empêcha de ces derniers d'attaquer en même temps les Français sur le front de Montréal. Dans les deux cas, l'auteur ne décide pas, il se contente de formuler des hypothèses comme s'il voulait créer un 
contrepoint entre l'accessoire (certains éléments du contexte) et l'essentiel (le récit lui-même). Dans les deux cas, l'embrayeur autoritaire "quoiqu'il en soit" fait obliquer le texte vers un terrain plus sûr: on passe de l'énonciation à l'énoncé ou, si l'on me permet ce parallélisme aventureux, de l'hypothèse à la Vérité.

Je ne serais pas surpris que ce balisage de la diégèse entre deux signaux aussi voyants ne fasse partie d'une stratégie historienne visant à rejeter à la périphérie l'accessoire pour laisser bonne place à l'événement central sur l'interprétation duquel ne subsiste aucun doute. Comme par hasard, l'auteur affiche la carence de sa documentation ou de sa mémoire seulement pour l'accessoire. Le procédé a le double avantage de régler une fois pour toutes le sort de l'inclassable, de l'inexplicable en le refoulant aux confins du récit et, par la grâce d'un aveu d'humilité, de rendre l'essentiel d'autant plus crédible.

Contrairement à ce qu'on pourrait croire à première vue, la fable ou la "conjecture", peuvent être accueillies, du moment qu'elles ne mettent pas en danger la cohérence globale de l'éconcé historique et qu'elles donnent à celui-ci le tremblement du vécu, de la pensée en action.

Me voici un peu loin, me direz-vous, de ma liste d'auteurs. Comme Charlevoix, je reviens à mon histoire. La fiction, ai-je dit, est le danger suprême. Comment en sortir? Jugeant les premiers auteurs de dictionnaires géographiques et historiques, Charlevoix écrit:

Ils n'avoient devant les yeux que peu de Memoires, dont les Auteurs se bornaient presque à rapporter ce qu'ils avoient vû, ou appris de témoins oculaires, et ne pouvoient quére être taxés que de quelque exaggeration.

On discerne bien ici le lieu commun de la source historique: la connaissance passe d'abord par le vu de témoins, mais doit devenir écrit (livre ou archive) pour servir à l'historien.

\section{La primauté du vu}

Comme j'ay plus de connessance de ces choses que beaucoup d'autres, j'en parles hardiment. . . J'ay eu le bonheur d'estre témoin oculeire de presque tout,

écrit Marie Morin au début de son Histoire simple et véritable, communément appelée Annales de l'Hôtel-Dieu de Montréal. Le témoin fiable est celui-là seul qui $a$ vu. Lafitau a vu de près les moeurs, les coutumes et la religion des Sauvages Amériquains; Sagard, présenté positivement, "raconte naïvement tout ce qu'il a vû," mais "il nous apprend peu de choses intéressantes" parce qu" il n'a pas eu le tems de voir assez bien les choses, encore moins de verifier tout ce qu'on lui avoit dit." On peut compter sur ce que Bacqueville de la Potherie "dit comme témoin oculaire," mais "il n'a pas toujours été bien instruit sur le reste." Est-ce à dire que l'acuité de la vision est liée à l'agir? Je le crois. En tout cas, plus le vu est greffé sur un faire important, plus le témoignage sera précieux, comme le manifestent les Jésuites de la Nouvelle-France "répandus dans toutes les nations" et "entrés" "dans toutes les affaires de la Colonie." Mais encore faut-il que cet agir soit transparent, animé d'intentions droites. 
Donc, primauté du vu, et méfiance du ouï-dire, comme chez Champlain. Le ouï-dire, c'est la voix anonyme collectivisée, la rumeur, la parole diffuse, fabulatrice, comme si la vue garantissait à elle seule la véracité. Charlevoix récuse davantage Hennepin et Lahontan en ne mentionnant pas leur qualité de témoin oculaire qu'en les attaquant violemment dans leurs thèses, leur comportement ou leur style. Voltaire, qui insistera tellement sur "l'incertitude de l'histoire," parlera de "cette certitude qui naît de la disposition unanime de mille témoins oculaires de différentes nations, sans que personne ait réclamé contre leur témoignage." (Article "Histoire" de l'Encyclopédie).

Si l'on se rappelle que la "Liste des Auteurs" se termine sur la mention "d'une aussi habile main," celle du cartographe Bellin, on se demandera si Charlevoix ne dépasse pas ici le cliché pour rejoindre une hantise de l'époque dont on verra des manifestations dix ans plus tard dans plusieurs articles et gravures de l'Encyclopédie: l'oeil et la main comme organes essentiels de saisie du réel. Je ne pense pas seulement ici à ces très belles planches représentant plusieurs dessins d'oeil ou de main, mais à d'autres mettant en scène un Monsieur bien habillé et très calme, montrant de sa main à une Dame le spectacle pas du tout effrayant d'un volcan dont la lave descend jusqu'à leurs pieds. Je pense à cette expression du médecin Tronchin que je choisis parce qu'elle n'est ni de d'Alembert, ni de Diderot, ni de Jaucourt: "l'inoculation confiée à des yeux éclairés et à des mains sages." Au départ, la fiabilité historique sera donc affaire d' acuité visuelle et de main-mise à la pâte de l'événement.

S'interroger sur le témoignage, c'est forcément s'interroger sur le témoin. On sait quelle importance y attachent les auteurs de l'Encyclopédie Diderot plus particulièrement:

Il faut considérer si les témoins sont oculaires ou non; ce qu'ils ont risqué pour se faire croire; quelle crainte ou quelles espérances ils avoient en annonçant aux autres des faits dont ils se disoient témoins oculaires: s'ils avoient exposé leur vie pour soûtenir leur déposition, il faut convenir qu'elle acquéreroit une grande force; que seroit-ce donc s'ils l'avoient sacrifiée et perdue? ${ }^{3}$

Détachée de son contexte, cette phrase semble un brin naïve; mais, faute de temps, je ne puis la replacer dans la discussion serrée à laquelle se livre Diderot sur la critique des sources historiques. Elle nous permet toutefois de mieux voir la position de Charlevoix. Celui-ci ne distingue pas comme Diderot, Voltaire, l'abbé de Prades, entre différents types de faits (simples/extraordinaires; transitoires/permanents; passés/présents; éloignés/rapprochés, etc.), entre différents types de témoins (oculaires, instruits, intéressés, nombreux, indépendants. . .); il ne pose le problème du témoin que par rapport à la contemporanéité de l'événement. Et encore, le témoin n'est accepté ou récusé que sur sa personne. Il s'agit moins de savoir si son témoignage est conditionné dans l'espace et le temps, limité par l'acuité de vision, la distance existentielle ou physique que d'établir d'abord la qualité de l'individu. Lahontan est récusé comme témoin en tant que "mauvais François" et "mauvais Chrétien," comme Hennepin qui aurait dû "se souvenir 
que c'étoit aux frais de la France, qu'il avoit voyagé dans l'Amérique, et que c'étoit au nom du Roy Très-Chrétien, que lui et le Sieur Dacan avoient pris possession des Pays, qu'ils avoient découverts." Il est symptomatique d'ailleurs que Charlevoix présente Lahontan comme un déclassé social, puis comme un chicanier qui subit la honte de la condamnation:

L'Auteur, quoi qu'homme de condition, fut d'abord Soldat en Canada. . . . il se brouilla avec le Gouverneur, fut cassé, et se retira . . .

Pourquoi Charlevoix attribue-t-il l'initiative de la brouille à Lahontan alors qu'il connaissait le naturel chicanier et autoritaire du Gouverneur Brouillan et savait que celui-ci avait été condamné pour concussion? Charlevoix aurait fort bien pu écrire quelque chose comme: "le Gouverneur et Lahontan, tout aussi mauvais sujets l'un que l'autre, se brouillèrent. . . ."

Mais la valorisation/dévalorisation du témoin est habituellement beaucoup plus subtile. Champlain "étoit habile Navigateur, homme de tête et de resolution, désintéressé, plein de zéle pour la Religion et pour l'Etat"; Sagard "paroît homme fort judicieux, et très-zélé, non-seulement pour le salut des ames, mais encore pour le progrès d'une Colonie." Comme par hasard, l'être accompagne un témoignage semblable à celui des Jésuites - donc fiable - et le paroître est attribué à qui ne voit pas de la même manière. Autre subtile distinction, que je note sans généraliser: Lescarbot est présenté favorablement, mais comme un "Auteur" qui "a ramassé tout ce qui avoit été écrit avant lui": "il paroît sincere, bien instruit, censé et impartial" (On sait que les témoignages de Champlain et Lescarbot ne concordent pas toujours).

Une dernière distinction, beaucoup plus importante celle-là, s'attache à la mise en écriture du témoignage. Voyons-en un exemple: celui du Récollet Leclercq. Ne parlons pas de la Nouvelle Relation de Gaspésie, jugée péremptoirement inintéressante parce que la géographie humaine et physique en est pauvre, alors que pourtant la "description" de la même région par Nicolas Denys est fort vantée. Le Premier Etablissement de la Foy est dévalorisé dès le départ parce qu'"on a lieu de croire que le Comte de Frontenac a mis la main" à "Cet Ouvrage." Jugement surprenant à un double titre:

1) Justifiant à priori son entreprise historique, Charlevoix avait écrit dans son "Projet" qu'une histoire de la Nouvelle-France n'exigeait pas que "toutes les parties" "soient de la même main."

2) Charlevoix s'en prend souvent aux faiseurs de "conjectures" qui suivent la mode, la voix pubique ou simplement leurs préjugés, plutôt que d'adopter une attitude désintéressée. Or, l'insinuation par le biais de la parole diffuse, du ouï-dire dont je parlais plus tôt, est fréquente chez Charlevoix:

Aussi passe-t-il pour constant que c'est Geudreville lui-même, qui a retouché la derniere Edition de ses Voyages [ceux de Lahontan].

Il paroît même qu'il [Hennepin] avoit défense de retourner dans l'Amérique, et que ce fut le chagrin, qu'il en conçut, qui le porta à s'en aller en Hollande, où il fit imprimer un troisiéme Ouvrage .... .

On leur [les Voyages de Lahontan] a rendu plus de justice en Canada, où l'Auteur passe communément pour un Romancier. 
Plus que mesquinerie ou concurrence dans le domaine du faire et de l'écrire, il faut retenir ici une conception de l'historien qui fait de celui-ci un analogue de Dieu, en ce qu'il sonde les reins et les coeurs, voit les ressorts cachés des actions les plus complexes, distingue sans possibilité d'erreur le vrai du faux, le sincère de l'apprêté; bref, il est le Créateur qui met ordre et lumière dans le Chaos.

\section{L'intermédiaire de l'écrit}

Un dernier point retiendra mon attention. Malgré la primauté du vu sur le oü̈-dire, du témoignage direct sur l'intermédiaire, l'historien n'est pas celui qui table sur le témoignage direct du témoin, mais sur la transcription de ce témoignage. Dans le corps de son ouvrage, Charlevoix ne confirme ni n'infirme un fait par ce qu'il a vu lui-même, mais par une autre pièce écrite. Pour être valable ou même utilisé, le témoignage devra franchir l'oralité et se fixer dans l'écriture, plus particulièrement le livre. Autrement dit, le seul regard retenu en définitive, n'est pas le regard de l'observateur, mais celui du lecteur: les premiers historiens et géographes "n'avoient devant les yeux que peu de Memoires dont les Auteurs se bornoient presque à rapporter ce qu'ils avoient vî, ou appris de témoins oculaires." Malgré toutes ces phrases sur l'observation visuelle, le regard critique n'est donc en définitive qu'un regard de liseur; le réel historique qu'on prétend représenter n'est pas le vu concret mais une vue de l'esprit fixée dans un livre.

Je dis un livre et non pas l'archive comme j'avais d'abord écrit, puisque Charlevoix oppose justement le livre à l'archive, non pour privilégier cette dernière, mais pour lui accorder un rôle d'appoint: l'archive n'apporte en effet que des "éclaircissemens" aux "Livres imprimés"; "les pièces originales" citées à la fin ne servent qu'à "remplir" les "vuides."

"Eclaircissemens", "vuides": ces mots peuvent encore nous éclairer sur ce qui constitue l'histoire chez Charlevoix. Dans les dépêches dẹs Gouverneurs et des Intendants, il a "puisé tout ce qui regarde le Gouvernement politique et militaire de la Nouvelle-France"; les mémoires de Perrot et Pénicault lui ont mieux fait comprendre les moeurs et coutumes des Indiens. C'est donc dire que ces deux sujets, moeurs et gouvernement, ne font pas fondamentalement partie de l'histoire. Se défendant d'avoir "voulu faire tomber" l'Histoire de Koempfer sur le Japon, Charlevoix affirme n'y avoir rien trouvé "pour l'historique" ("j'aurois bien eu de la peine à en tirer une feüille d'impression") même s'il contient "une Notice fort ample de cet Empire, qui renferme le Gouvernement, la Police, la Religion, la Géographie et le Commerce des Hollandois" (Avertissement). Ce resserrement du champ historique était présent dès le départ puisque dans son "Projet" de 1735, Charlevoix prévoyait disposer les données recueillies en deux groupes: celles qui, organiquement liées entre elles, hiérarchisées en système, constitueraient le corps de l'histoire et celles qui, simultanées dans la réalité, risqueraient d'interférer avec la séquence narrative. L'ordonnance même de l'Histoire publiée en 3 volumes va exactement dans le même sens.

T.I: - "Avertissement"

- Histoire et description. . . ., L.I-XIII, p. 1-571. 
- "Particularités de la vie et de la mort de quelques sauvages chrétiens", pp. 572-600.

_ "Table des matières"

T.II: $\quad-$ Histoire et description. ., L.XIII-XXII, pp. 1-502.

- "Table des matières", pp. 503-582.

- "Description des plantes principales de l'Amérique septentrionale", paginé de 1 à 56 .

T.III: - "Remarques de M. Bellin . . . sur des Cartes et des plans ...., p. I-XIX.

- "Dissertation préliminaire sur l'origine des Amériquains", p. $1-43$.

- Journal historique. . . p. 44-500.

- "Projet d'un corps d'histoires du Nouveau Monde", p. I-IV.

- "Fastes chronologiques du Nouveau Monde. . .", p. V-XL

- "Liste et examen des auteurs. . .", p. XLI-XLII.

Malgré que son auteur en ait contre le roman, l'Histoire et description est en réalité construite comme un roman sagement disposé en chapitres chronologiques. Tout ce qui ne touche pas à l'aventure collective du groupe colonisateur, tout ce que Charlevoix ne peut narrativiser (faune, commerce, culture matérielle) est habituellement refoulé en dehors des bornes strictes du récit. Pour le Diderot et le Voltaire de l'Encyclopédie, on s'en souvient, l'opération historique était une entreprise de totalisation du savoir sur tous les aspects du passé humain.

Je me résume. Les gloses de Charlevoix sur les auteurs utilisés ne relèvent pas d'un simple commentaire bibliographique. Elles mettent en cause une conception de l'histoire dont on peut maintenant tracer les grandes lignes, en s'inspirant de l'“Avertisssement" et du "Projet d'un corps d'Histoires . . . ." Tout d'abord, il faut distinguer l'historique de l'énoncé historique: ou, si l'on préfère, d'une part, la diégèse, c'est-à-dire l'ensemble des éléments événementiels retenus, et d'autre part, le récit, c'est-à-dire l'organisation linguistique, l'arrangement textuel qui permet de représenter le réseau diégétique.

Lettré cultivé, professeur et rédacteur au Journal de Trévoux, Charlevoix définit l'histoire par rapport aux grands genres littéraires comme l'épopée, la tragédie et la comédie avec lesquels elle entretient une parenté par le sujet traité, aussi bien que par les personnages mis en scène. Mais là même où elle prétend s'en éloigner, elle en demeure assez près malgré tout: les sources où elle puise sont essentiellement écrites (le livre, le papier d'archive, le "bon mémoire"). Ne devient source que ce qui a été bien "digéré" une première fois par un mémorialiste ou un scripteur quelconque et une seconde fois par l'historien. On est loin de l'entreprise encyclopédique où un Voltaire, un Boucher d'Argis, un Diderot interrogent, outre les sources utilisées pour l'Histoire et description, la tradition orale, les textes de lois, les "monuments" 
(médailles aussi bien que statues et édifices), les manifestations de la culture matérielle (artefacts, logement, outils, habillement) et spirituelle (Beaux-Arts et littérature, langue, religion, contes, légendes et chansons populaires. . .). Diderot était évidemment tout disposé à accueillir les sources les plus diverses, puisque dans sa propre ville de Langres (cf. article du même nom) des découvertes archéologiques et une sauvegarde systématique de "monuments" ont permis de déterminer qu'une colonie romaine avait existé, qu'on y avait présenté du théâtre, que les Gaulois vénéraient Pluton, etc.

Plus spécifiquement, l'Histoire de la Nouvelle-France ne sera pas celle d'un "grand Empire" avec ses "révolutions importantes" et ses personnages célèbres; elle s'apparente plutôt à la comédie qui, prenant "ses sujets" et "ses Acteurs dans la vie privée," n'offre rien d'éclatant mais "des graces naïves, beaucoup de varieté et de simplicité, une sage distribution." Bien à regret, semble-t-il, Charlevoix se voit presque condamné à relater l'évolution d'une colonie qui n'offre rien de palpitant: des personnages sans beaucoup de panache, des "détails peu interessans en soi," retenus seulement pour leur exotisme ("pays éloignés").

On peut même se demander si Charlevoix n'a pas eu l'impression de se livrer à un pensum:

"Pour venir au sujet de l'Ouvrage, que je présente aujourd'hui au Public, j'en connois tous les desavantages. Il s'agit d'un pays immense, et qui après plus de deux Siécles, qui se sont écoulés depuis que nous l'avons découvert, est encore moins peuplé qu'il ne l'étoit alors, quoi qu'il y ait passé assez de François pour remplacer au triple les Sauvages qu'on y trouva, et qu'on ne puisse pas leur reprocher de les avoir détruits. Cela n'annonce point une Histoire remplie de faits interessans; mais on la demandoit cette Histoire, et on avoit raison de la demander. C'est celle de toutes les Colonies Françoises du nouveau Monde, qui ont été honorées du titre de la nouvelle France, ou qui en ont fait partie; et elle nous manquoit." (Avertissement)

Dans le même "Avertissement" Charlevoix écrit encore:

Il m'auroit sans doute été plus aisé et plus agréable de ne prendre, si j'ose ainsi m'exprimer, que la crême de l'Histoire du nouveau Monde. J'aurois été bien-tôt à la fin de ma carriere, et j'aurois eu apparemment plus de Lecteurs....

En réalité, l'histoire de la Nouvelle-France, semblable à celle de SaintDominique publiée plus tôt est l'histoire d'un échec, comme l'expriment clairement les dernières lignes de l'"Avertissement."

Qui a donc arrêté le progrès de l'Evangile parmi ces Barbares, et d'où vient que la plus ancienne de nos Colonies, celle qui naturellement devoit se peupler davantage, est encore la moins puissante de toutes?

Mince consolation de savoir que cette histoire "ne présente, au moins dans l'origine du principal Etablissement, que des objets capables de faire estimer notre Nation, la seule, qui ait eu le secret de gagner l'affection des Amériquains." 
Restent donc les "détails curieux," sur lesquels insiste tellement Charlevoix et qui, par leur pointillisme, forment une sorte d"'Histoire suivie" capable "d'interesser et d'instruire." Mais n'allons pas croire que la surabondance des détails fasse perdre l'ensemble de vue. L'entreprise historique est d'abord une opération sélective, classificatoire ensuite: "on pourra avoir une connaissance entiere de chaque region du Nouveau Monde . . . de ce qui s'y est passé de considerable . . .; de ce qu'elle renferme de plus curieux." Charlevoix veut rivaliser avec les Voyages et Mémoires pour l'abondance et la curiosité des détails, mais sauvegarder l'ordre, la précieuse unité d'ensemble. Une métaphore revient souvent chez lui: celle de la noyade:

L'Histoire du Nouveau Monde ne sera plus en danger de périr par sa propre abondance; les choses, qui sont véritablement dignes de la curiosité des Lecteurs, n'y seront plus noyées dans les inutilités, pour ne rien dire de plus, ni embarassées dans les contradictions ...

("Projet d'un corps d'histoire. ...)

Surabondance du détail, on le voit, mais surabondance maîtrisée, mise en ordre, passée à l'aune de la vérité. Car la mise en ordre, finalement, conduit à la vérité. Si elle peut accueillir les éléments les plus incongrus ou surprenants, la vérité exclut les contradictions. Une vérité dont on serait bien embarrassé de délimiter les contours et encore davantage les critères. Charlevoix écrit:

nous avons encore des regles certaines de critique pour distinguer les Pieces legitimes et authentiques, de ce nombre prodigieux d'Ecrits hazardés, dont la plûpart altérent la vérité jusqu'au point de la rendre méconnaissable /. . . . . . il nous reste encore un rayon de lumiere, à la faveur duquel nous pouvons dégager la vérité de ce monstrueux amas de fables, qui l'ont presqu'entierement éclipsée. . .

("Projet d'un corps d'histoires. ...)

Mais on ne saura jamais en définitive d'où vient ce rayon de lumière, ni quelles sont ces règles certaines.

Le même mise en ordre, la même ordonnance générale devra prendre en charge l'énoncé historique. N'ayant pas le temps d'entrer dans le détail, je rappelle seulement quelques éléments. V. LeBlanc parle de la NouvelleFrance "d'une manière confuse, peu exacte, et sans ordre." Si l'on se rappelle que la grande faute de Lahontan, de Hennepin, de Leclercq était la boursouflure, la déclamation et l'embarras, on pensera aux règles de clarté, de simplicité, d'hiérarchisation des éléments de la phrase de l'idéal classique. Il faudrait encore ajouter une dernière remarque: si l'on franchit les limites de la phrase, si l'on déborde la stylistique, on trouve la vieille catégorie de la disposition: pas seulement celle des éléments verbaux en eux-mêmes mais celle touchant la distribution de la matière factuelle: dans l'Avertissement, Charlevoix parle de "l'ordre et la distribution des faits" comme d'un "vaste champ à la Critique."

Réal Ouellet Université Laval 


\section{Notes}

1 "Projet d'un corps d'histoires du Nouveau-Monde", Publié d'abord dans le Journal de Trévoux, en janvier 1735, et repris à la fin du T. III de L'Histoire et description de la Nouvelle-France (Paris: Nyon Fils, 1744). Les "Liste et examen des auteurs que j'ai consultés pour composer cet ouvrage" sont aussi publiés aux T. III, tandis que l'“Avertissement," auquel je me référerai parfois est placé en tête du T. III. L'Histoire et description parut en 1744 chez cinq éditeurs différents et sous deux formats: in quarto en 3 volumes et duodecimo en 6 volumes. Je citerai ici l'édition Nyon Fils en 3 volumes. C'est celle que reproduisent anastatiquement les Editions Elysée (Montréal, 1976).

${ }^{2}$ Fr.-X. Garneau, "Préface," Histoire du Canada (Montréal: Beauchemin et Valois, T. I, 1889), pp. VI-VII.

3 Article "Agnus Scythicus" de l'Encyclopédie. Cf. aussi, parmi beaucoup d'autres: "certitude", "critique", "fait", "histoire"... . 